\title{
Wolf Search Algorithm for Solving Optimal Reactive Power Dispatch Problem
}

\author{
K. Lenin*, B. Ravindhranath Reddy, M. Surya Kalavathi \\ Jawaharlal Nehru Technological University Kukatpally, Hyderabad 500 085, India \\ email: gklenin@gmail.com*
}

\begin{abstract}
This paper presents a new bio-inspired heuristic optimization algorithm called the Wolf Search Algorithm (WSA) for solving the multi-objective reactive power dispatch problem. Wolf Search algorithm is a new bio - inspired heuristic algorithm which based on wolf preying behaviour. The way wolves search for food and survive by avoiding their enemies has been imitated to formulate the algorithm for solving the reactive power dispatches. And the speciality of wolf is possessing both individual local searching ability and autonomous flocking movement and this special property has been utilized to formulate the search algorithm. The proposed (WSA) algorithm has been tested on standard IEEE 30 bus test system and simulation results shows clearly about the good performance of the proposed algorithm.
\end{abstract}

Keywords: modal analysis, optimal reactive power, transmission loss, wolf search algorithm

\section{Introduction}

Optimal reactive power dispatch problem is subject to number of uncertainties and at least in the best case to uncertainty parameters given in the demand and about the availability equivalent amount of shunt reactive power compensators. Optimal reactive power dispatch plays a major role for the operation of power systems, and it should be carried out in a proper manner, such that system reliability is not got affected. The main objective of the optimal reactive power dispatch is to maintain the level of voltage and reactive power flow within the specified limits under various operating conditions and network configurations. By utilizing a number of control tools such as switching of shunt reactive power sources, changing generator voltages or by adjusting transformer tap-settings the reactive power dispatch can be done. By doing optimal adjustment of these controls in different levels, the redistribution of the reactive power would minimize transmission losses. This procedure forms an optimal reactive power dispatch problem and it has a major influence on secure and economic operation of power systems. Various mathematical techniques like the gradient method [1, 2] Newton method [3]and linear programming [4-7] have been adopted to solve the optimal reactive power dispatch problem. Both the gradient and Newton methods has the difficulty in handling inequality constraints. If linear programming is applied then the input- output function has to be expressed as a set of linear functions which mostly lead to loss of accuracy. The problem of voltage stability and collapse play a major role in power system planning and operation [8]. Enhancing the voltage stability, voltage magnitudes within the limits alone will not be a reliable indicator to indicate that, how far an operating point is from the collapse point. The reactive power support and voltage problems are internally related to each other. This paper formulates by combining both the real power loss minimization and maximization of static voltage stability margin (SVSM) as the objectives. Global optimization has received extensive research attention, and a great number of methods have been applied to solve this problem. Evolutionary algorithms such as genetic algorithm have been already proposed to solve the reactive power flow problem $[9,10]$. Evolutionary algorithm is a heuristic approach used for minimization problems by utilizing nonlinear and non-differentiable continuous space functions. In [11], by using Genetic algorithm optimal reactive power flow has been solved, and the main aspect considered is network security maximization. In [12] is proposed to improve the voltage stability index by using Hybrid differential evolution algorithm. In [13] Biogeography Based algorithm proposed to solve the reactive power dispatch problem. In [14] a fuzzy based method is used to solve the optimal reactive power scheduling method and it minimizes real power loss and maximizes Voltage Stability Margin. In [15] an improved evolutionary programming is used to solve the optimal 
reactive power dispatch problem. In [16] the optimal reactive power flow problem is solved by integrating a genetic algorithm with a nonlinear interior point method. In [17] a standard algorithm is used to solve ac-dc optimal reactive power flow model with the generator capability limits. In [18] proposed a two-step approach to evaluate Reactive power reserves with respect to operating constraints and voltage stability. In [19] a programming based proposed approach used to solve the optimal reactive power dispatch problem. In [20] is presented a probabilistic algorithm for optimal reactive power provision in hybrid electricity markets with uncertain loads. This research paper proposes a new bio-inspired heuristic search optimization algorithm, the Wolf Search Algorithm (WSA), for solving the optimal reactive power dispatch problem and this algorithm is based on wolf preying behaviour activity. Algorithm possesses both individual local searching ability and autonomous flocking movement. Wolf hunts independently by remembering its own trait and it will merge with its peer when the peer is in better position. The swarming behaviour of WSA has more advantage than that of algorithms like PSO [21], Fish [22] and Firefly [23]. WSA functions as multiple leaders swarming from multiple directions to reach the best solution, rather than searching as a single flock. How the wolf jumps far out of its hunter's visual range to avoid being trapped like that algorithm design will jump away from the local optimal solution. The wolves in the nature have best memory capability for they can hide food in caches; also they sense and track down a prey from distances of miles away. They themselves do set markers in their territory in various methods like by urinating at the borders. Researcher Sebastian Vetter and his team, from the University of Vienna have been studying the high level of observational spatial memory in the wolf. Main assumption is that the wolves are functioning as searching agents in the WSA optimization algorithm are empowered by memory caches that can able to store the previously visited various positions. The proposed algorithm WSA been evaluated in standard IEEE 30 bus test system \& the simulation results shows that our proposed approach outperforms all reported algorithms in minimization of real power loss and voltage stability index.

\section{Voltage Stability Evaluation}

\subsection{Modal Analysis for Voltage Stability Evaluation}

Modal analysis is one among best methods for voltage stability enhancement in power systems. The steady state system power flow equations are given by.

$\left[\begin{array}{l}\Delta \mathrm{P} \\ \Delta \mathrm{Q}\end{array}\right]=\left[\begin{array}{cc}\mathrm{J}_{\mathrm{p} \theta} & \mathrm{J}_{\mathrm{pv}} \\ \mathrm{J}_{\mathrm{q} \theta} & \mathrm{J}_{\mathrm{QV}}\end{array}\right]$

Where

$\Delta \mathrm{P}=$ Incremental change in bus real power.

$\Delta Q=$ Incremental change in bus reactive

Power injection

$\Delta \theta=$ incremental change in bus voltage angle.

$\Delta \mathrm{V}=$ Incremental change in bus voltage Magnitude

$J_{p \theta}, J_{P V}, J_{Q \theta}, J_{Q V}$ jacobian matrix are the sub-matrixes of the System voltage stability is affected by both $P$ and $Q$.

To reduce (1), let $\Delta P=0$, then:

$$
\begin{aligned}
& \Delta \mathrm{Q}=\left[\mathrm{J}_{\mathrm{QV}}-\mathrm{J}_{\mathrm{Q} \theta} \mathrm{J}_{\mathrm{P} \theta^{-1}} \mathrm{~J}_{\mathrm{PV}}\right] \Delta \mathrm{V}=\mathrm{J}_{\mathrm{R}} \Delta \mathrm{V} \\
& \Delta \mathrm{V}=\mathrm{J}^{-1}-\Delta \mathrm{Q}
\end{aligned}
$$

Where,

$\mathrm{J}_{\mathrm{R}}=\left(\mathrm{J}_{\mathrm{QV}}-\mathrm{J}_{\mathrm{Q} \theta} \mathrm{J}_{\mathrm{P} \theta^{-1}} \mathrm{JPV}\right)$

$\mathrm{J}_{\mathrm{R}}$ is called the reduced Jacobian matrix of the system. 
2.2. Modes of Voltage Instability

Voltage Stability characteristics of the system have been identified by computing the Eigen values and Eigen vectors.

Let,

$\mathrm{J}_{\mathrm{R}}=\xi \wedge \eta$

Where,

$\xi=$ right eigenvector matrix of $J_{R}$

$\eta=$ left eigenvector matrix of $J_{R}$

$\Lambda=$ diagonal eigenvalue matrix of $J_{R}$ and

$\mathrm{J}_{\mathrm{R}^{-1}}=\xi \wedge^{-1} \eta$

From (5) and (8), we have:

$\Delta \mathrm{V}=\xi \wedge^{-1} \eta \Delta \mathrm{Q}$

Or,

$$
\Delta \mathrm{V}=\sum_{\mathrm{I}} \frac{\xi_{\mathrm{i}} \eta_{\mathrm{i}}}{\lambda_{\mathrm{i}}} \Delta \mathrm{Q}
$$

Where $\xi_{i}$ is the ith column right eigenvector, and $\eta$ is the ith row left eigenvector of JR. $\lambda_{i}$ is the ith Eigen value of $J_{R}$.

The ith modal reactive power variation is:

$\Delta \mathrm{Q}_{\mathrm{mi}}=\mathrm{K}_{\mathrm{i}} \xi_{\mathrm{i}}$

Where,

$\mathrm{K}_{\mathrm{i}}=\sum_{\mathrm{j}} \xi_{\mathrm{ij}}{ }^{2}-1$

Where

$\xi_{\mathrm{ji}}$ is the jth element of $\xi_{\mathrm{i}}$

The corresponding ith modal voltage variation is:

$\Delta \mathrm{V}_{\mathrm{mi}}=\left[1 / \lambda_{\mathrm{i}}\right] \Delta \mathrm{Q}_{\mathrm{mi}}$

If $\left|\lambda_{\mathrm{i}} \quad\right|=0$ then the ith modal voltage will collapse. Then:

In (10), let $\Delta Q=e_{k}$ where $e_{k}$ has all its elements zero except the kth one being 1 .

$$
\Delta V=\sum_{\mathrm{i}} \frac{\eta_{1 \mathrm{k}} \xi_{1}}{\lambda_{1}}
$$

$\eta_{1 \mathrm{k}} \mathrm{k}$ th element of $\eta_{1}$

$\mathrm{V}-\mathrm{Q}$ sensitivity at bus $\mathrm{k}$

$$
\frac{\partial \mathrm{V}_{\mathrm{K}}}{\partial \mathrm{Q}_{\mathrm{K}}}=\sum_{\mathrm{i}} \frac{\eta_{1 \mathrm{k}} \xi_{1}}{\lambda_{1}}=\sum_{\mathrm{i}} \frac{\mathrm{P}_{\mathrm{ki}}}{\lambda_{1}}
$$

\section{Problem Formulation}

The objectives of the reactive power dispatch problem is to minimize the system real power loss and maximize the static voltage stability margins (SVSM).

\subsection{Minimization of Real Power Loss}

Minimization of the real power loss (Ploss) in transmission lines is mathematically stated as follows. 


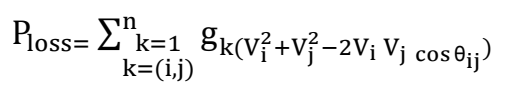

Where $\mathrm{n}$ is the number of transmission lines, $\mathrm{gk}$ is the conductance of branch $\mathrm{k}, \mathrm{Vi}$ and $\mathrm{Vj}$ are voltage magnitude at bus $\mathrm{i}$ and bus $\mathrm{j}$, and $\theta \mathrm{ij}$ is the voltage angle difference between bus $\mathrm{i}$ and bus j.

\subsection{Minimization of Voltage Deviation}

Minimization of the voltage deviation magnitudes (VD) at load buses is mathematically stated as follows.

$$
\text { Minimize VD }=\sum_{\mathrm{k}=1}^{\mathrm{nl}}\left|\mathrm{V}_{\mathrm{k}}-1.0\right|
$$

Where $\mathrm{nl}$ is the number of load busses and $\mathrm{Vk}$ is the voltage magnitude at bus $\mathrm{k}$.

\subsection{System Constraints}

Objective functions are subjected to these constraints shown below.

Load flow equality constraints:

$$
\begin{aligned}
& P_{G i}-P_{D i}-V_{i \sum_{j=1}^{n b} V_{j}}\left[\begin{array}{cc}
G_{i j} & \cos \theta_{i j} \\
+B_{i j} & \sin \theta_{i j}
\end{array}\right]=0, i=1,2 \ldots, n b \\
& Q_{G i}-Q_{D i}-V_{i \sum_{j=1}^{n b} V_{j}}\left[\begin{array}{cc}
G_{i j} & \cos \theta_{i j} \\
+B_{i j} & \sin \theta_{i j}
\end{array}\right]=0, i=1,2 \ldots, n b
\end{aligned}
$$

Where, $n b$ is the number of buses, $P_{G}$ and $Q_{G}$ are the real and reactive power of the generator, $P_{D}$ and $Q_{D}$ are the real and reactive load of the generator, and $G_{i j}$ and $B_{i j}$ are the mutual conductance and susceptance between bus $i$ and bus $j$.

Generator bus voltage $\left(\mathrm{V}_{\mathrm{Gi}}\right)$ inequality constraint:

$V_{G i}^{\min } \leq V_{G i} \leq V_{G i}^{\max }, i \in n g$

Load bus voltage $\left(\mathrm{V}_{\mathrm{Li}}\right)$ inequality constraint:

$\mathrm{V}_{\mathrm{Li}}^{\min } \leq \mathrm{V}_{\mathrm{Li}} \leq \mathrm{V}_{\mathrm{Li}}^{\max }, \mathrm{i} \in \mathrm{nl}$

Switchable reactive power compensations $\left(\mathrm{Q}_{\mathrm{Ci}}\right)$ inequality constraint:

$\mathrm{Q}_{\mathrm{Ci}}^{\min } \leq \mathrm{Q}_{\mathrm{Ci}} \leq \mathrm{Q}_{\mathrm{Ci}}^{\max }, \mathrm{i} \in \mathrm{nc}$

Reactive power generation $\left(Q_{G i}\right)$ inequality constraint:

$\mathrm{Q}_{\mathrm{Gi}}^{\min } \leq \mathrm{Q}_{\mathrm{Gi}} \leq \mathrm{Q}_{\mathrm{Gi}}^{\max }, \mathrm{i} \in \mathrm{ng}$

Transformers tap setting $\left(\mathrm{T}_{\mathrm{i}}\right)$ inequality constraint:

$\mathrm{T}_{\mathrm{i}}^{\min } \leq \mathrm{T}_{\mathrm{i}} \leq \mathrm{T}_{\mathrm{i}}^{\max }, \mathrm{i} \in \mathrm{nt}$

Transmission line flow $\left(\mathrm{S}_{\mathrm{Li}}\right)$ inequality constraint:

$\mathrm{S}_{\mathrm{Li}}^{\min } \leq \mathrm{S}_{\mathrm{Li}}^{\max }, \mathrm{i} \in \mathrm{nl}$

Where, nc, ng and nt are numbers of the switchable reactive power sources, generators and transformers.

IJEEI Vol. 3, No. 1, March 2015: $7-15$ 


\section{Wolf Search Algorithm}

Wolves are social predators that hunt in packs and uses stealth when hunting prey together. In behaviour of ants it utilizes pheromones to communicate with their peers to know about food source. WSA [24] also do this kind of communication, which decreases the run time of the search. Wolves are unique, partially cooperative characteristics and usually move in a group in coupled formation, but have tendency to take down the prey individually. WSA naturally balances scouting the problem space in random groups and individual. During hunting, wolves will group themselves as they approach their prey. This peculiar characteristic prompts the searching agents in WSA to move for a better position, like the same way wolves continuously change their positions for better ones. When hunting, wolves search for prey and also keenly watch the threats from hunters or other animals like tigers etc. Each wolf in the pack chooses its own way \& position continuously moving to a better state for the prey and also for threats in all directions. When wolves' bumping into their enemies it is well equipped with a threat probability and it dashes a great distance away from its present position. The same way in WSA avoids the deadlock of getting trapped in local optimal solution. The direction and distance the wolf moving away from a threat are random, and is similar to mutation and crossover in Genetic algorithm .Wolves have very high sense of smell and it can easily locate prey by scent. Similarly, in the WSA each wolf has a sensing distance that creates visual distance. This visual distance is applied to search the global optimum and in moving to a better position and for jumping out of visual range. In search mode, the wolves are move in Brownian motion (BM), which imitates the random drifting of particles suspended in fluid.

\section{Basic logics of wolf search}

There are three rules that act as basic logics of the Wolf Search Algorithm (WSA)

Rule 1: Each wolf has visual area as a fixed one and with a radius defined by $v$ for $X$ as a set of continuous possible solutions. Each wolf can sense companions who are all appear within its visual circle. The footstep expanse by which the wolf moves at a time is normally smaller than its visual distance.

Rule 2: The fitness of the objective function represents the wolf's current position. If there is more options the wolf will chose the best terrain inhabited by another wolf from the given options. If not, the wolf will continue to move randomly in BM.

Rule 3: if the wolf will sense an enemy then the wolf will immediately escape to a random position far from the threat and beyond its visual range.

WSA implementation in based on the fitness of the objective function and it reflects the quality of a terrain position which will eventually lead to food.

Wolf often changes in position in search of food and also to safeguard form the enemies. Wolf trust with other wolves in movement because they never prey each other. The movement done by one wolf will be watched by other wolves simultaneously and they position themselves in chance of finding food also with care of them by continuously moving. If the current wolf's location is greater the distance of the companion location, then that new location will be less attractive one even though the new position may be good one. Wolf's willingness to move is decreased means, and then that movement will obey the inverse square law. The formula is $(r)=\frac{I_{0}}{r^{2}}$, where lo is the origin of food and $r$ is the distance between the food or we can denote that distance between the new terrain and the wolf.

This is the similar formula in the firefly algorithm, for the calculation of attractiveness. The incentive formula for the wolf search by using absorption coffeicient and gaussian equation, can be written as:

$$
\beta(r)=\beta_{o} e^{-r^{2}}
$$

Normally all the wolves want to move better position based on colonized by their peers position and it depends on many factors like visual distance and how the initial wolf covers the area. Wolf will visualize the other wolves location each other i.e. it will compare the range of distance and set by itself in best position for preying and also from enemies. The movement can be written as:

$$
x(i)=x(i)+\beta_{o} e^{-r^{2}}(x(j)-x(i))+\operatorname{escape}()
$$


Where, escape ( $)$ is a function that calculates a random position to jump to with a constraint of minimum length; $v, x$ is the wolf, which represents a candidate solution; and $x(j)$ is the peer with a better position as represented by the value of the fitness function. The second term of the above equation represents the change in value or gain achieved by progressing to the new position. $r$ is the distance between the wolf and its peer with the better location.

There are three types of preying that takes place in sequence,

1) Preying initiatively

Wolf feed on prey it represents the optimization function as objective. By using the visual boundary wolf will have step by step movement on constantly seeing the prey and it will have random movement from the current step to forward or backward depending on the prey position. If it thinks particular position as best one then it will omit other wolves movements. Then it will move in own direction.

\section{2) Prey passively}

In passive mode the wolf will compare the position with its peers and will improve the current position. Wolf will move to passive mode when its own movement does not find food or insecurity for its movement.

\section{3) Escape}

Wolves normally have enemies in nature and threat will be there always. If any threat is found, it will relocate very quickly form the current position to new position which will be normally greater distance than that of the normal visual range. This can be written in equation as:

$$
\text { if moving }=\left\{\begin{array}{l}
x(i)=x(i)+\alpha \cdot r \cdot \operatorname{rand}() \text { prey } \\
x(i)=x(i)+\alpha \cdot s \cdot \text { escape }() \text { escape }
\end{array}\right.
$$

Where $x(i)$ is the wolf's location; $a$ is the velocity; $v$ is the visual distance; rand() is a random function whose mean value distributed in $[-1,1], s$ is the step size, which must be smaller than $v$; and escape() is a custom function that randomly generates a position greater than $v$ and less than half of the solution boundary.

Wolf algorithm for solving optimal reactive power dispatch problem:

Step 1: Objective function $f(x), x=\left(x_{1}, x_{2}, . . x d\right)^{\top}$

Step 2: Initialize the population, $x_{i}(i=1,2, . ., W)$

Step 3: initialize parameters

$r=$ radius of the visual range

$\mathrm{s}=$ step size by which a wolf moves at a time

$\alpha=$ velocity factor of wolf

$\mathrm{pa}=\mathrm{a}$ user-defined threshold [0-1], determines how often foe appears

Step 4: WHILE ( $\mathrm{t}<$ generations and also for stopping criteria is not met)

step5: FOR $\mathrm{i}=1$ : $\mathrm{W} / /$ each wolf

step6: Prey new food initiatively ();

step7: Generation of new location ();

step8: To check whether the next location suggested by the random number generator is new one.

step8: If not, repeat generating random location.

Step9:IF(dist $\left(x_{i}, x_{j}\right)<r$ and $x_{j}$ is better as $\left.f\left(x_{i}\right)<f\left(x_{j}\right)\right) x_{i}$ moves towards $x_{j} / / x_{j}$ is a better than $x_{i}$

Step 10: ELSE IF

$\mathrm{x}_{\mathrm{i}}=$ Prey new food passively ();

Step 11: END IF

Generation of new location ();

IF (rand ()>pa)

$x_{i}=x_{i}+\operatorname{rand}()+v$; wolf escape to a new position.

END IF

END FOR

END WHILE

IJEEI Vol. 3, No. 1, March 2015 : 7 - 15 


\section{Simulation Results}

The accurateness of the proposed WSA method is demonstrated by testing it on standard IEEE-30 bus system. The IEEE-30 bus system has 6 generator buses, 24 load buses and 41 transmission lines of which four branches are (6-9), (6-10), (4-12) and (28-27) - are with the tap setting transformers. The lower voltage magnitude limits at all buses are 0.95 p.u. and the upper limits are 1.1 for all the PV buses and 1.05 p.u. for all the PQ buses and the reference bus. The simulation results have been presented in Tables 1, 2, $3 \& 4$. And in the Table 5 shows the proposed algorithm powerfully reduces the real power losses when compared to other given algorithms. The optimal values of the control variables along with the minimum loss obtained are given in Table 1. Corresponding to this control variable setting, it was found that there are no limit violations in any of the state variables.

Table 1. Results of WSA - ORPD optimal control variables

\begin{tabular}{cc}
\hline Control variables & Variable setting \\
\hline V1 & 1.041 \\
V2 & 1.042 \\
V5 & 1.041 \\
V8 & 1.031 \\
V11 & 1.002 \\
V13 & 1.040 \\
T11 & 1.00 \\
T12 & 1.00 \\
T15 & 1.02 \\
T36 & 1.01 \\
Qc10 & 3 \\
Qc12 & 3 \\
Qc15 & 4 \\
Qc17 & 0 \\
Qc20 & 3 \\
Qc23 & 4 \\
Qc24 & 4 \\
Qc29 & 3 \\
Real power loss & 4.3209 \\
SVSM & 0.2462 \\
\hline
\end{tabular}

Table 2. Results of WSA -Voltage Stability Control Reactive Power Dispatch Optimal Control Variables

\begin{tabular}{cc}
\hline Control Variables & Variable Setting \\
\hline V1 & 1.043 \\
V2 & 1.043 \\
V5 & 1.042 \\
V8 & 1.031 \\
V11 & 1.006 \\
V13 & 1.034 \\
T11 & 0.090 \\
T12 & 0.090 \\
T15 & 0.090 \\
T36 & 0.090 \\
Qc10 & 4 \\
Qc12 & 4 \\
Qc15 & 3 \\
Qc17 & 3 \\
Qc20 & 0 \\
Qc23 & 3 \\
Qc24 & 2 \\
Qc29 & 3 \\
Real power loss & 4.9870 \\
SVSM & 0.2471 \\
\hline
\end{tabular}

ORPD together with voltage stability constraint problem was handled in this case as a multi-objective optimization problem where both power loss and maximum voltage stability margin of the system were optimized simultaneously. Table 2 indicates the optimal values of these control variables. Also it is found that there are no limit violations of the state variables. It indicates the voltage stability index has increased from 0.2462 to 0.2471 , an advance in the 
system voltage stability. To determine the voltage security of the system, contingency analysis was conducted using the control variable setting obtained in case 1 and case 2 . The Eigen values equivalents to the four critical contingencies are given in Table 3 . From this result it is observed that the Eigen value has been improved considerably for all contingencies in the second case.

Table 3. Voltage Stability under Contingency State

\begin{tabular}{cccc}
\hline SI.No & Contingency & $\begin{array}{c}\text { ORPD } \\
\text { Setting }\end{array}$ & $\begin{array}{c}\text { VSCRPD } \\
\text { Setting }\end{array}$ \\
\hline 1 & $28-27$ & 0.1410 & 0.1427 \\
2 & $4-12$ & 0.1658 & 0.1668 \\
3 & $1-3$ & 0.1774 & 0.1784 \\
4 & $2-4$ & 0.2032 & 0.2047 \\
\hline
\end{tabular}

Table 4. Limit Violation Checking Of State Variables

\begin{tabular}{|c|c|c|c|c|}
\hline \multirow{2}{*}{$\begin{array}{c}\text { State } \\
\text { variables }\end{array}$} & \multicolumn{2}{|c|}{ limits } & \multirow{2}{*}{ ORPD } & \multirow{2}{*}{ VSCRPD } \\
\hline & Lower & upper & & \\
\hline Q1 & -20 & 152 & 1.3422 & -1.3269 \\
\hline Q2 & -20 & 61 & 8.9900 & 9.8232 \\
\hline Q5 & -15 & 49.92 & 25.920 & 26.001 \\
\hline Q8 & -10 & 63.52 & 38.8200 & 40.802 \\
\hline Q11 & -15 & 42 & 2.9300 & 5.002 \\
\hline Q13 & -15 & 48 & 8.1025 & 6.033 \\
\hline V3 & 0.95 & 1.05 & 1.0372 & 1.0392 \\
\hline V4 & 0.95 & 1.05 & 1.0307 & 1.0328 \\
\hline V6 & 0.95 & 1.05 & 1.0282 & 1.0298 \\
\hline V7 & 0.95 & 1.05 & 1.0101 & 1.0152 \\
\hline V9 & 0.95 & 1.05 & 1.0462 & 1.0412 \\
\hline V10 & 0.95 & 1.05 & 1.0482 & 1.0498 \\
\hline V12 & 0.95 & 1.05 & 1.0400 & 1.0466 \\
\hline V14 & 0.95 & 1.05 & 1.0474 & 1.0443 \\
\hline V15 & 0.95 & 1.05 & 1.0457 & 1.0413 \\
\hline V16 & 0.95 & 1.05 & 1.0426 & 1.0405 \\
\hline V17 & 0.95 & 1.05 & 1.0382 & 1.0396 \\
\hline V18 & 0.95 & 1.05 & 1.0392 & 1.0400 \\
\hline V19 & 0.95 & 1.05 & 1.0381 & 1.0394 \\
\hline V20 & 0.95 & 1.05 & 1.0112 & 1.0194 \\
\hline V21 & 0.95 & 1.05 & 1.0435 & 1.0243 \\
\hline V22 & 0.95 & 1.05 & 1.0448 & 1.0396 \\
\hline V23 & 0.95 & 1.05 & 1.0472 & 1.0372 \\
\hline V24 & 0.95 & 1.05 & 1.0484 & 1.0372 \\
\hline V25 & 0.95 & 1.05 & 1.0142 & 1.0192 \\
\hline V26 & 0.95 & 1.05 & 1.0494 & 1.0422 \\
\hline V27 & 0.95 & 1.05 & 1.0472 & 1.0452 \\
\hline V28 & 0.95 & 1.05 & 1.0243 & 1.0283 \\
\hline V29 & 0.95 & 1.05 & 1.0439 & 1.0419 \\
\hline V30 & 0.95 & 1.05 & 1.0418 & 1.0397 \\
\hline
\end{tabular}

Table 5. Comparison of Real Power Loss

\begin{tabular}{lc}
\hline \multicolumn{1}{c}{ Method } & Minimum loss \\
\hline Evolutionary programming [25] & 5.0159 \\
Genetic algorithm [26] & 4.665 \\
Real coded GA with Lindex as SVSM [27] & 4.568 \\
Real coded genetic algorithm [28] & 4.5015 \\
Proposed WSA method & 4.3209 \\
\hline
\end{tabular}

\section{Conclusion}

In this paper, the WSA has been successfully implemented to solve optimal reactive power dispatch (ORPD) problem. The main advantages of WSA when applied to the ORPD problem is optimization of different type of objective function, i.e real coded of both continuous and discrete control variables, and without difficulty in handling nonlinear constraints. The proposed WSA algorithm has been tested on the IEEE 30-bus system. Simulation Results clearly show the good performance of the proposed algorithm in reducing the real power loss and enhancing the voltage profiles within the limits. 


\section{References}

[1] O Alsac, B Scott. Optimal load flow with steady state security. IEEE Transaction. 1973; 745-751.

[2] Lee KY, Paru YM, Oritz JL. A united approach to optimal real and reactive power dispatch. IEEE Transactions on power Apparatus and systems. 1985; 104: 1147-1153

[3] A Monticelli, MVF Pereira, S Granville. Security constrained optimal power flow with post contingency corrective rescheduling. IEEE Transactions on Power Systems:PWRS-2. 1987; 1: 175-182.

[4] Deeb N, Shahidehpur SM. Linear reactive power optimization in a large power network using the decomposition approach. IEEE Transactions on power system. 1990; 5(2): 428-435.

[5] E Hobson. Network consrained reactive power control using linear programming. IEEE Transactions on power systems PAS -99. 1980; (4): 868-877.

[6] KY Lee, YM Park, JL Oritz. Fuel -cost optimization for both real and reactive power dispatches. IEE Proc. 131C(3): 85-93.

[7] MK. Mangoli, KY Lee. Optimal real and reactive power control using linear programming. Electr.Power Syst.Res. 1993; 26: 1-10.

[8] CA Canizares, ACZ de Souza, VH Quintana, Comparison of performance indices for detection of proximity to voltage collapse. 1996; 11(3): 1441-1450.

[9] SR Paranjothi, K Anburaja. Optimal power flow using refined genetic algorithm. Electr.Power Compon.Syst. 2002; 30: 1055-1063.

[10] D Devaraj, B Yeganarayana. Genetic algorithm based optimal power flow for security enhancement. IEE proc-Generation Transmission and Distribution. 2005; 152.

[11] A Berizzi, C Bovo, M Merlo, M Delfanti. A ga approach to compare orpf objective functions including secondary voltage regulation. Electric Power Systems Research. 2012; 84(1): 187-194.

[12] CF Yang, GG Lai, CH Lee, CT Su, GW Chang. Optimal setting of reactive compensation devices with an improved voltage stability index for voltage stability enhancement. International Journal of Electrical Power and Energy Systems. 2012; 37(1): 50-57.

[13] P Roy, S Ghoshal, S Thakur. Optimal var control for improvements in voltage profiles and for real power loss minimization using biogeography based optimization. International Journal of Electrical Power and Energy Systems. 2012; 43(1): 830-838.

[14] B Venkatesh, G Sadasivam, M Khan. A new optimal reactive power scheduling method for loss minimization and voltage stability margin maximization using successive multi-objective fuzzy Ip technique. IEEE Transactions on Power Systems. 2000; 15(2): $844-851$.

[15] W Yan, S Lu, D Yu. A novel optimal reactive power dispatch method based on an improved hybrid evolutionary programming technique. IEEE Transactions on Power Systems. 2004; 19(2): 913 - 918.

[16] W Yan, F Liu, C Chung, K Wong. A hybrid genetic algorithminterior point method for optimal reactive power flow. IEEE Transactions on Power Systems. 2006; 21(3): 1163 -1169.

[17] J Yu, W Yan, W Li, C Chung, K Wong. An unfixed piecewiseoptimal reactive power-flow model and its algorithm for ac-dc systems. IEEE Transactions on Power Systems. 2008; 23(1): 170 -176.

[18] F Capitanescu. Assessing reactive power reserves with respect to operating constraints and voltage stability. IEEE Transactions on Power Systems. 2011; 26(4): 2224-2234.

[19] Z Hu, X Wang, G. Taylor. Stochastic optimal reactive power dispatch: Formulation and solution method. International Journal of Electrical Power and Energy Systems. 2010; 32(6): 615 - 621.

[20] A Kargarian, M Raoofat, M Mohammadi. Probabilistic reactive power procurement in hybrid electricity markets with uncertain loads. Electric Power Systems Research. 2012; 82(1): 68 - 80.

[21] XS Yang, S Deb, S Fong. Accelerated Particle Swarm Optimization and Support Vector Machine for Business Optimization and Applications. The Third International Conference on Networked Digital Technologies (NDT 2011), Springer CCIS 136. Macau. 2011; 53-66.

[22] Y Peng. An Improved Artificial Fish Swarm Algorithm for Optimal Operation of Cascade Reservoirs. Journal of Computers. 2011; 6(4): 740-746.

[23] XS Yang. Firefly algorithms for multimodal optimization. Stochastic Algorithms: Foundations and Applications. Lecture Notes in Computer Sciences. 2009; 169-178.

[24] Rui Tang, Simon Fong, Xin-She Yang, Suash Deb. Wolf Search Algorithm with Ephemeral Memory. Seventh International Conference on Digital Information Management, ICDIM 2012. Macao. 2012.

[25] Wu QH, Ma JT. Power system optimal reactive power dispatch using evolutionary programming. IEEE Transactions on power systems. 1995; 10(3): 1243-1248.

[26] S Durairaj, D Devaraj, PS Kannan. Genetic algorithm applications to optimal reactive power dispatch with voltage stability enhancement. IE(I) Journal-EL. 2006; 87.

[27] D Devaraj. Improved genetic algorithm for multi - objective reactive power dispatch problem. European Transactions on electrical power. 2007; 17: 569-581.

[28] P Aruna Jeyanthy, Dr D Devaraj. Optimal Reactive Power Dispatch for Voltage Stability Enhancement Using Real Coded Genetic Algorithm. International Journal of Computer and Electrical Engineering. 2010; 2(4): 1793-8163. 